\title{
Obtenção de scaffolds bioabsorvíveis a partir de blenda de PLGA e IR com adição de hidroxiapatita
}

\author{
Obtaining bioabsorable scaffolds from PLGA \\ and IR blend with the addition of hydroxyap- \\ atite
}

Pâmella Schramm Oliveira ${ }^{1}$, Luiz Fernando Rodrigues Junior ${ }^{1}$, Tiago Moreno Volkmer ${ }^{2}$, Nayrim Brizuela Gerra ${ }^{3}$, Luís Alberto Loureiro dos Santos ${ }^{3}$

\footnotetext{
${ }^{1}$ Engenharia Biomédica EngBio/UFN, CEP: 7015 97010-032, Santa Maria, Rio Grande do Sul, Brasil.

${ }^{2}$ Engenharia de Materiais EngMat/UFN, CEP: 7015 97010-032, Santa Maria, Rio Grande do Sul, Brasil.

${ }^{3}$ Laboratório de Biomateriais - LABIOMAT - PPGE3M/UFRGS, CEP: 15051 91501-970, Porto Alegre, Rio Grande do Sul, Brasil.

e-mail: schramm.pso@gmail.com, luiz.fernando@unifra.br, tiagovolkmer@gmail.com,nayrimb@gmail.com, luis.santos@ufrgs.br
}

\section{RESUMO}

A obtenção de scaffolds bioativos a partir da blenda polimérica de poli (ácido láctico-co-glicólico) (PLGA) e poliisopreno (IR) com diferentes adições de Hidroxiapatita (HAp) é proposta como uma alternativa à engenharia de tecidos. Para o preparo das soluções poliméricas, a blenda de PLGA/IR e a HAp foram dissolvidas em clorofórmio. Posteriormente, o scaffold foi processado por electrospinning, variando-se a distância entre a seringa e o coletor. As amostras foram caracterizadas quanto a composição química e estrutural por potencial zeta, difração de raios-X (DRX) e espectroscopia de infravermelho por transformada de Fourier (FTIR). Para avaliação da morfologia usou-se a microscopia eletrônica de varredura (MEV) e para o comportamento citotoxicológico utilizou-se a metodologia de analises de viabilidade celular. O valor de potencial zeta da HAp foi de $-22 \mathrm{mV}$ e o FTIR e DRX foram condizentes com a literatura. As imagens de MEV permitiram a análise do tamanho médio e desvio padrão das fibras obtidas, comprovando uma diferença significativa entre as amostras. $\mathrm{O}$ ensaio de citotoxicidade mostrou que o produto obtido é biocompatível, porém as amostras com menor formação de fibras são menos biocompatíveis. Os resultados demonstraram a ampla influência da distância entre o coletor e a ponta da seringa e da relação blenda/HAp, sendo que o scaffold obtido com a maior distância coletor/seringa e maior relação HAp/blenda apresentou fibras com tamanho médio menores e maior homogeneidade e baixa citotoxicidade celular.

Palavras-chave: Engenharia de tecidos, electrospinning, PLGA/IR.

\section{ABSTRACT}

The aim of this paper is the obtainment of bioactive scaffolds from poly (lactic acid-co-glycolic acid) (PLGA) and polyisoprene (IR) polymer blends with different additions of hydroxyapatite (HAp) as an alternative for tissue engineering. For the preparation of the polymer solutions, the PLGA/IR blend and the HAp were dissolved in chloroform. Subsequently, the scaffold was processed by electrospinning, varying the distance between the syringe and the collector. Scaffolds chemical and phase composition were characterized by zeta potential, X-ray diffraction (XRD) and Fourier transform infrared spectroscopy (FTIR). Scanning electron microscopy (SEM) was used to evaluate the morphology and for the cytotoxic behavior the cellular viability analysis methodology was used. The zeta potential value of the HAp was $-22 \mathrm{mV}$ and the FTIR and XRD were consistent with the literature. The SEM images allowed the analysis of the mean size and standard deviation of the obtained fibers, demonstrating a significant difference among the samples. The results showed the great influence of the distance between the collector and the tip of the syringe and the blend/HAp ratio, and the scaffold obtained with the largest collector/ syringe distance and the highest HAp/blend ratio showed fibers with a smaller mean size and greater homogeneity and low cellular cytotoxicity.

Keywords: Tissue engineering, electrospinning, PLGA/IR. 


\section{INTRODUÇÃO}

A engenharia de tecidos tem como objetivo auxiliar na regeneração e restauração dos tecidos deformados ou lesados, gerando substitutos biológicos capazes de reparar, manter ou aperfeiçoar o desempenho e atividade dos mesmos [1]. Dentre as possibilidades de desenvolvimento na área de engenharia de tecidos, pode-se citar a criação de scaffolds bioativos que proporcionem uma liberação local de fatores de crescimento, influenciando no tratamento tecidual [2]. Para tal, a seleção dos biomateriais é uma das etapas de maior importância, visto que, diferentes tipos de biomateriais irão apresentar distintos comportamentos mecânicos, físicoquímicos e de degradabilidade [3].

Entre as técnicas que visam produzir scaffolds com propriedades adequadas para a engenharia de tecidos, a técnica de electrospinning ou eletrofiação tem como vantagens a produção de nanofibras naturais ou sintéticas, com elevada área superficial e poros com diâmetros controlados que variam de nanômetros a micrômetros [4][5]. Além disso, é um processo barato que pode ser facilmente ampliado através da utilização de várias fieiras e é surpreendentemente versátil, pois quase todos polímeros solúveis podem ser processados em nanofibras [6].

O cis-1,4-poliisopreno, polímero natural conhecido como poli-isopreno (IR), tem poucas aplicações como biomaterial, porém aplicações cirúrgicas previamente estudadas indicam que a presença desse material induz a regeneração celular e neovascularização [7][8].

O poli (ácido láctico-co-glicolico) (PLGA) é um dos mais utilizados polímeros bioreabsorvíveis. Trata-se de um copolímero de ésteres alifáticos, que tem sido amplamente utilizado como implantes que degradam e são absorvidos pelo organismo [9][10][11]. Contudo, trata-se de um polímero que apresenta comportamento frágil, o que pode inviabilizar a sua utilização em dispositivos que necessitem de altos índices de deformação plástica [12][8].

Misturas ou blendas tratam-se de uma abordagem que representa uma das áreas com mais rápido crescimento na ciência dos polímeros e têm como objetivo obter características novas através da combinação de propriedades de polímeros distintos [13][14]. Como exemplo dessas, um recente estudo visando a manipulação das propriedades mecânicas do PLGA obteve uma blenda entre este e o IR, a qual foi registrada com o nome de blenda Cellprene ${ }^{\circledR}$ e teve patente solicitada em 2011. A partir dos resultados positivos deste estudo, surge a possibilidade de aplicação do Cellprene ${ }^{\circledR}$ em engenharia de tecidos [10][8].

As biocerâmicas, como os fosfatos de cálcio ou hidroxiapatita (HAp), apresentam excelente biocompatibilidade, bioatividade, ausência de toxicidade, taxas de degradação variáveis e osteocondutividade [15]. Entretanto, têm seu uso limitado devido à baixa resistência e baixa tenacidade quando comparadas com o osso humano [16]. Uma combinação de um biopolímero ou blenda polimérica e uma biocerâmica poderia melhorar as propriedades mecânicas, aumentar a estabilidade da degradação e afinidades celulares dos componentes individuais. Assim, a incorporação de biocerâmicas em uma matriz de polímero nanofibroso, não apenas mimetizaria a estrutura óssea natural, mas também melhoraria as propriedades mecânicas e a resposta biológica dos scaffods [17].

O presente trabalho teve como objetivo a obtenção de scaffolds bioativos com matriz polimérica de PLGA e IR, com carga de hidroxiapatita, pela metodológica de electrospinning. A viabilidade para a aplicação de tal scaffold em engenharia de tecidos foi avaliada através do estudo da influência de diferentes adições de hidroxiapatita, variação da distância entre o coletor e a ponta da seringa, caracterização química, estrutural, morfológica e de citotoxicidade.

\section{MATERIAIS E MÉTODOS}

\subsection{Materiais}

Neste trabalho, foram utilizados os seguintes materiais: blenda Cellprene ${ }^{\circledR}$ fornecida pelo LABIOMAT- laboratório de Biomateriais e Cerâmicas Avançadas da Universidade Federal do Rio Grande do Sul e produzida conforme Marques [10], com uma relação de PLGA/IR de (60/40); Hidroxiapatita fornecida pela empresa Aldrich, peneirada em peneira \#325; solvente clorofórmio (Synth) conforme recebido.

\subsection{Obtenção de Scaffolds}

Para o preparo das soluções poliméricas dissolveu-se primeiramente as blendas PLGA/IR em clorofórmio e posteriormente a adição de diferentes proporções de HAp, utilizando uma placa de agitação em velocidade 6 . Ao estar completamente homogênea a solução, esta foi transferida para uma seringa de $10 \mathrm{~mL}$ com agulha de 
dimensão 1,20 x 40mm. O agitador mecânico foi mantido em velocidade constante de $500 \mathrm{rpm}$, e assim, as amostras foram preparadas a partir da variação da distância entre a solução polimérica e o coletor. A Tabela 1 apresenta os valores de polímero, solvente, voltagem, distância e HAp que foram utilizados nas diferentes formulações.

Tabela 1: Relação das diferentes variáveis estudadas para o desenvolvimento dos Scaffolds.

\begin{tabular}{ccccc}
\hline AMOSTRAS & $\begin{array}{c}\text { VOLUME DE } \\
\text { SOLVENTE }(\mathbf{m L})\end{array}$ & $\begin{array}{c}\text { TENSÃO } \\
(\mathbf{k V})\end{array}$ & $\begin{array}{c}\text { DISTÂNCIA } \\
(\mathbf{c m})\end{array}$ & $\begin{array}{c}\text { HAp } \\
(\%)\end{array}$ \\
\hline D5H1 & 10 & 15 & 5 & 1 \\
D5H3 & 10 & 15 & 5 & 3 \\
D5H5 & 10 & 15 & 5 & 5 \\
D7H1 & 10 & 15 & 7 & 1 \\
D7H3 & 10 & 15 & 7 & 3 \\
D7H5 & 10 & 15 & 7 & 5 \\
\hline
\end{tabular}

\subsection{Caracterização}

A estabilidade da suspensão foi analisada pela medição de Potencial Zeta, determinado por meio de suspensão de amostra de HAp com solução de $\mathrm{NaCl}(0,01 \mathrm{M}$, Synth), onde esta foi alocada em um porta amostra modelo DTS1070. A análise foi realizada em temperatura de $25^{\circ} \mathrm{C}$, através da submissão da amostra a um campo elétrico no equipamento Zetasizer Nano-Zs, da Malvern.

A difração de raios-X (DRX) verificou se a fase hidroxiapatita estava presente nas amostras obtidas, utilizando um difratômetro de raios-X Bruker, modelo D2 PHASER, equipado com ânodo de cobre (radiação $\mathrm{K} \alpha \mathrm{Cu}, \lambda=1.5406 \AA$ ) operado a $30 \mathrm{kV}$ e $10 \mathrm{~mA}$. Os parâmetros usados nas análises foram: um intervalo angular de $20^{\circ}<2 \theta<45^{\circ}$ com passos de $0,02^{\circ}$ por um período de 1 s para cada passo.

O ensaio de Espectroscopia de Infravermelho por Transformada de Fourier (FTIR) foi utilizado para avaliação e caracterização dos grupamentos químicos da HAp, blenda polimérica e scaffold obtido. Para o preparo em cada caso, foram feitas pastilhas de $\mathrm{KBr}$ contendo aproximadamente $\mathrm{x} \mathrm{mg}$ de amostra. O equipamento utilizado foi o espectrômetro PerkinElmer, modelo Spectro One.

A Microscopia Eletrônica de Varredura (MEV) foi utilizada para avaliar a morfologia dos scaffolds e os tamanhos de fibras obtidas. O equipamento usado foi um microscópio Phenom, modelo ProX, com tensão de aceleração de $10 \mathrm{kV}$ e para evitar o carregamento das amostras, as mesmas foram revestidas com ouro.

Para avaliação da citotoxicidade, células de linhagem celular VERO foram semeadas em uma concentração de 1 x 105 células/poço em placas de 96 poços, sendo cultivadas em estufa de $5 \%$ de $\mathrm{CO} 2$ à $37^{\circ} \mathrm{C}$ durante 24 horas, para o experimento 3-(4,5) dimetiltialzolil -2,5 difeniltetrazólio (MTT). A técnica foi baseada na metabolização do reagente MTT (cor amarela), em cristais de formazan (cor violácea). A reação ocorreu através da atividade da enzima mitocondrial succinato-desidrogenase, a qual permanece ativa somente nas células viáveis. Assim, foi possível estabelecer parâmetros de citotoxicidade e taxas de proliferação celular [18].

As amostras dos scaffolds foram esterilizadas por luz UV por 30 minutos e mantidas em cabine de fluxo circular a fim de evitar contaminação externa no período entre esterilização e cultivo. As amostras foram alocadas em placa de cultura, as células foram tratadas e após 24 horas de tratamento foi adicionado em cada poço $20 \mu \mathrm{L}$ de MTT ( $5 \mathrm{mg} / \mathrm{mL}$ ), diluído em tampão fosfato salino (PBS 1X). A placa foi homogeneizada levemente e novamente mantida em estufa de $\mathrm{CO} 2(5 \%)$ à $37^{\circ} \mathrm{C}$, por 3 horas. Após o sobrenadante foi retirado e adicionou-se $200 \mu \mathrm{L}$ de dimetilsulfóxido (DMSO). A placa foi novamente agitada e a leitura realizada em leitora de ELISA no comprimento de onda de $570 \mathrm{~nm}$.

\section{RESULTADOS E DISCUSSÕES}

\subsection{CARACTERIZAÇÃO QUÍMICA E ESTRUTURAL}

A HAp analisada apresentou um potencial zeta de valor médio de $-22,6 \mathrm{mV}$. Sendo este o valor esperado, pois acredita-se que potencial zeta de valor negativo estimula a adsorção de íons $\mathrm{Ca}^{2+}$ que participam na deposição da matriz extracelular (osteocalcina e osteopontina) necessária para a adesão celular [19][20][21]. 
Na Figura 2 é apresentado o difratograma da HAp. O ensaio de DRX identificou picos característicos da HAp hexagonal, como os picos assinalados no difratograma que correspondem à ficha padrão de difração de número 09-0432 do International Center for Diffraction Data - ICDD.

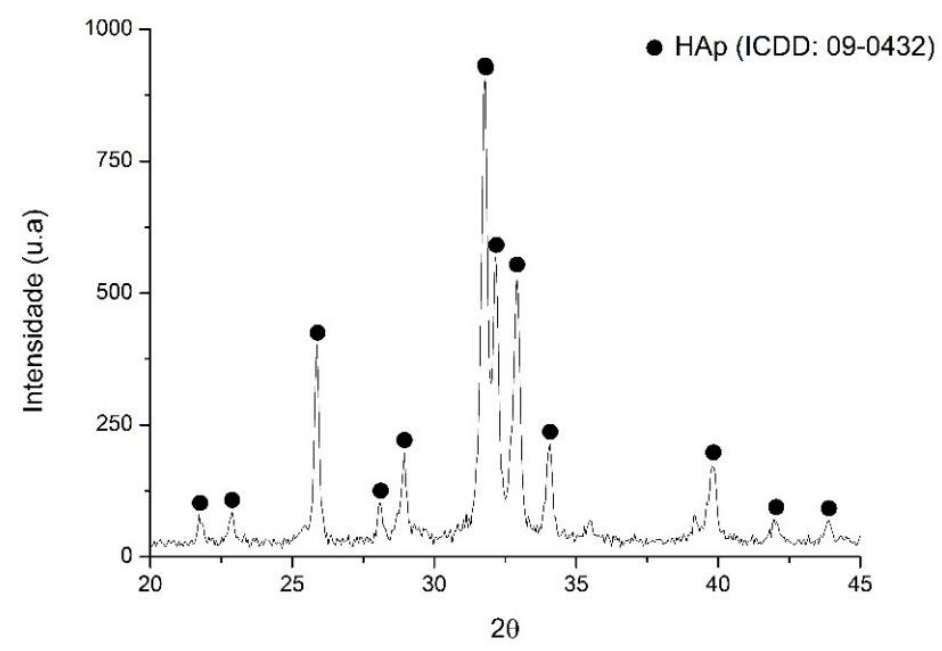

Figura 1: Difratograma de raios-X da HAp.

As análises dos grupamentos químicos presentes na HAp, Blenda Cellprene® (PLGA/IR) e Scaffold (Blenda polimérica + HAp) foram realizados por meio de espectroscopia de infravermelho (Figura 3). A partir das estruturas moleculares previamente conhecidas, nos espectros da Blenda Cellprene® e do Scaffold observa-se bandas relativas ao grupamento $\mathrm{C}=\mathrm{C}$, condizente ao IR e bandas relativas ao grupamento $\mathrm{C}=\mathrm{O}$, condizentes ao PLGA. Além disso, bandas características de estiramentos simétricos e assimétricos dos grupos $\mathrm{CH}_{2}$ e $\mathrm{CH}_{3}$ entre 2980-2850 $\mathrm{cm}^{-1}$, condizentes ao IR e PLGA [22][23]. Assim como, as bandas relativas ao grupamento $\mathrm{PO}_{4}{ }^{3-}$, características da HAp, são observadas nos espectros da HAp e do Scaffold, confirmando assim a presença desta no scaffold obtido.



Figura 2: Espectro de infravermelho da Blenda Cellprene®, HAp e Scaffold de D5H5 (blenda Cellprene® + HAp). 


\subsection{CARACTERIZAÇÃO MORFOLÓGICA}

As imagens adquiridas por Microscopia Eletrônica de Varredura possibilitaram a caracterização morfológica dos scaffolds obtidos pela técnica de electrospinning. As micrografias dos scaffolds são apresentadas na Figura 3.

As Figuras 3(A-C) referem-se as micrografias dos scaffolds D5H1, D5H3 e D7H1, respectivamente. Observa-se que não houve formação de fibras nas estruturas das amostras com menores relações de HAp/blenda e distância scaffold. O scaffold de maior distância e adição de $1 \%$ de HAp também não formou fibras na estrutura.

As Figuras 3(E e F) apresentam as micrografias das amostras D5H5 e D7H5, sendo estas com 5\% de adição de HAp e distâncias de $7 \mathrm{~cm}$ e $5 \mathrm{~cm}$, respectivamente. Nota-se que em ambos os casos ocorre a formação de fibras porosas, pois provavelmente a solução se tornou termodinamicamente instável durante a evaporação do solvente e assim, as fibras de electrospinning se separaram em fases ricas e pobres em polímero, de tal modo que a última fase acabou gerando porosidades nas fibras [24].

A partir do software Image $J$ foi realizado a medida de 12 fibras das amostras D7H5 e D5H5. Os valores das médias e desvios padrão do diâmetro são apresentados na Figura 4. O teste ANOVA One-Way, utilizando Tukey para comparação entre as médias, confirmou que há uma diferença significativa entre elas ( $\mathrm{p}=0,00258)$. Percebe-se então, que o aumento da distância tendeu a diminuir o tamanho médio das fibras e formou uma estrutura mais homogênea, pois o desvio padrão de D7H5 é menor que D5H5. Na amostra utilizando menor distância, o tempo de vôo do elemento de fluido diminuiu e provavelmente o solvente não evaporou-se totalmente no caminho coletor-agulha, tornando o diâmetro das fibras irregulares [25][4][5].

As Figuras 3(D e F) mostram as micrografias dos scaffolds com adição de 3\% e 5\% de HAp e distância de $7 \mathrm{~cm}$, respectivamente. Os valores das médias e desvios padrão do diâmetro são apresentados na Figura 5. O teste ANOVA One-Way, utilizando Tukey para comparação entre as médias, confirmou que há uma diferença significativa entre elas $(p=0,004934)$. Portanto, nota-se que o aumento de proporção de HAp adicionada influência na diminuição do tamanho da fibra e aumenta a homogeneidade da estrutura da amostra, conforme constatado pelos valores de desvio padrão obtidos. Resultados semelhantes foram observados em estudos anteriores e provavelmente a redução do diâmetro das fibras está relacionado a alteração da viscosidade e condutividade elétrica da solução. Além disso, com o aumento da HAp na solução, ocorre a aglomeração de nanopartículas e a presença de tais agregados na ponta da agulha pode ter diminuído o diâmetro efetivo do orifício da agulha, levando a formação de fibras mais finas [26][27].
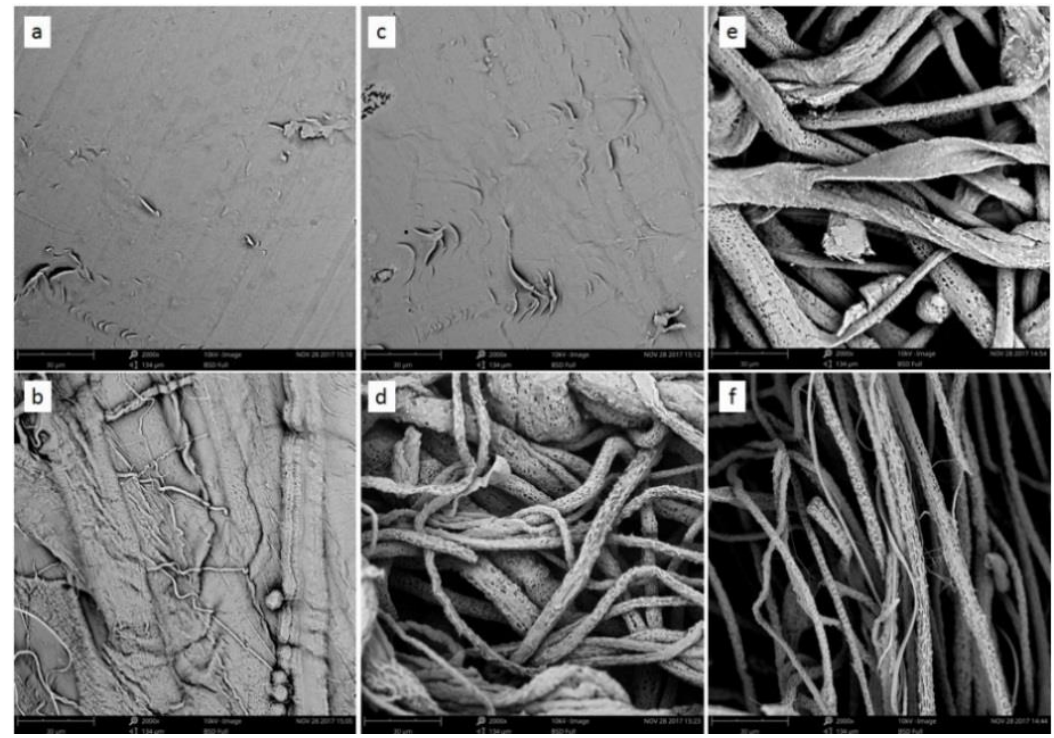

Figura 3: Micrografias mostrando a morfologia dos scaffolds de (a) D5H1, (b) D7H1, (c) D5H3, (d) D7H3, (e) D5H5 e (f) D7H5, com aumento de 2000x. 


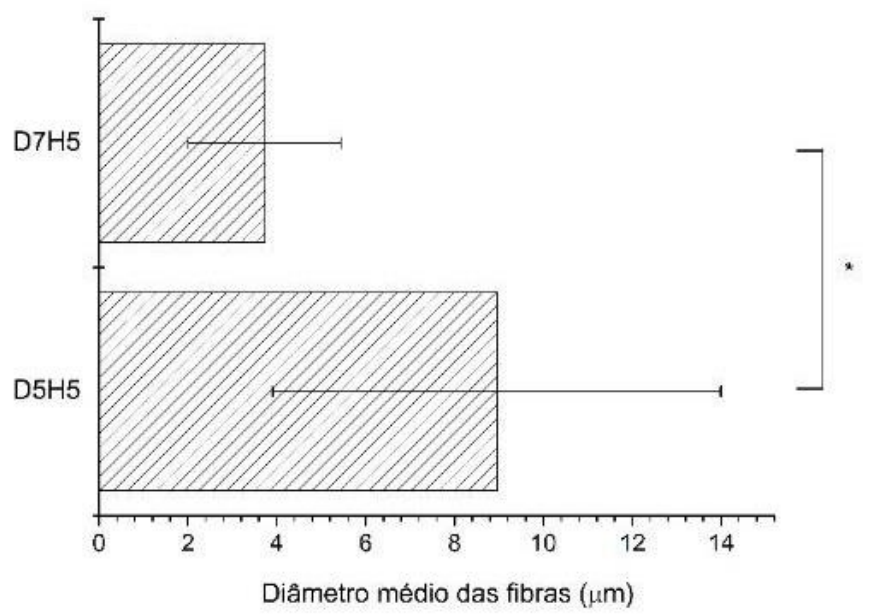

Figura 4: Média e desvio padrão do tamanho das fibras de D5H5 e D7H5. Os valores foram calculados com n=12 e medidas das fibras obtidas pelo software Image $J$. $* p=0,00258$

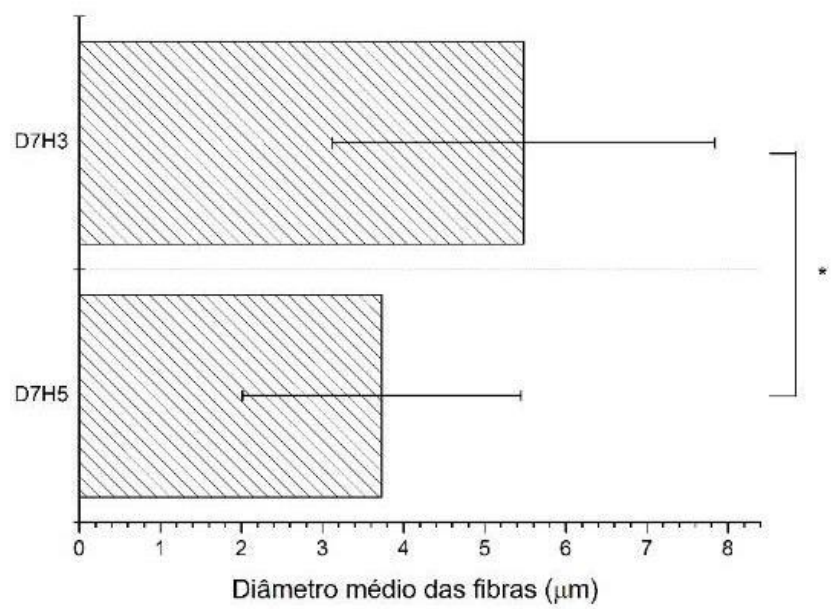

Figura 5: Média e desvio padrão do tamanho das fibras de D7H3 e D7H5. Os valores foram calculados com n=12 e medidas das fibras obtidas pelo software Image J. * p=0,004934.

\subsection{CITOTOXICIDADE}

Os resultados das médias do ensaio de viabilidade celular pelo método MTT são apresentados na Figura 6. Nota-se que foi possível a obtenção de materiais biocompatíveis, sendo o de resultado mais satisfatório o da amostra D7H5, de maior distância coletor/seringa e da relação HAp/blenda. Visto que todas as amostras tratavam-se do mesmo material, a maior citotoxicidade da amostra D5H5 provavelmente ocorreu devido à presença do solvente na solução, pois este provavelmente não evaporou-se totalmente no caminho coletoragulha [25][4][5]. 


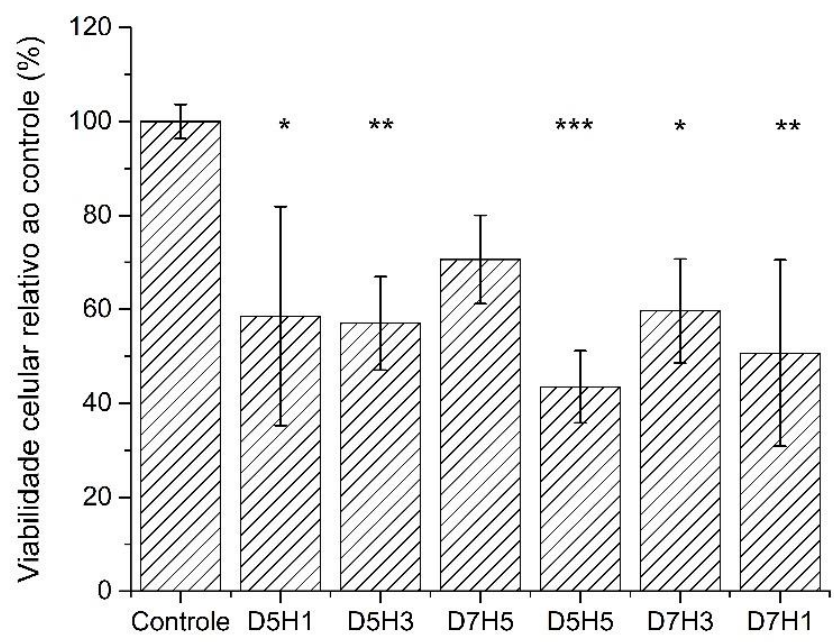

Figura 6: Avaliação da citotoxicidade dos scaffolds obtidos pela técnica de electrospinnig.

\section{AGRADECIMENTOS}

\section{UFN e LABIOMAT.}

\section{CONCLUSÃO}

A metodologia utilizada permitiu a obtenção de scaffolds bioativos com matriz polimérica de PLGA e IR, com carga de hidroxiapatita, pelo método de electrospinning A HAp utilizada para a confecção dos scaffolds obteve valor de potencial zeta conforme esperado, assim como o espectro e difratograma de raios-X. O espectro da blenda Cellprene ${ }^{\circledR}$ apresentou bandas relativas aos grupamentos de PLGA e IR, confirmando a presença deles na estrutura. O scaffold D5H5 obtido pela técnica de electrospinnig foi analisado por espectroscopia de infravermelho e apresentou bandas relativas aos grupamentos $\mathrm{C}=\mathrm{C}, \mathrm{C}=\mathrm{O}, \mathrm{CH}_{2}$ e $\mathrm{CH}_{3}$, condizentes a blenda Cellprene ${ }^{\circ}$, bem como, apresentou bandas relativas ao grupamento $\mathrm{PO}_{4}{ }^{3-}$, condizentes a estrutura de HAp. A partir das imagens de MEV dos scaffolds, conclui-se que o aumento da distância e presença da HAp diminuiu o tamanho médio das fibras, assim como, aumentou a homogeneidade da estrutura. O teste de viabilidade celular constatou que os scaffolds obtidos não são citotóxicos.

\section{BIBLIOGRAFIA}

[1] TABATA, Y. "Biomaterial technology for tissue engineering applications", Journal of The Royal Society Interface. v. 6, n. 3, p. 311-324. 2009.

[2] CARTMELL, S. "Controlled Release Scaffolds for Bone Tissue Engineering”, Journal of Pharmaceutical Science. v. 98, n. 2, p. 430-441. 2009.

[3] MONTEIRO, N. D. S. "Caracterização de matrizes de quitosano para a regeneração de tecidos produzidas pela técnica de TIPS”, Dissertação de M.Sc., Faculdade de Ciências e Tecnologia, Universidade Nova de Lisboa, Lisboa, 2008.

[4] BHARDWAJ, N., KUNDU, S. C. Electrospinning: A fascinating fiber fabrication technique. Biotechnology Advances. v. 28, n. 3, pp. 325-347. 2010.

[5] SILL, T. J., RECUM, H. A. V. "Electrospinning: Applications in drug delivery and tissue engineering", Biomaterials. v. 29, n. 13, p. 1989-2006. 2008.

[6] CHEW, S., WEN, Y., DZENIS, Y., et al., "The Role of Electrospinning in the Emerging Field of Nanomedicine”, Current Pharmaceutical Design. v. 12, n. 36, p. 4751-4770. 2006.

[7] CHEN, Q., LIANG, S., THOUAS, G. A. "Elastomeric biomaterials for tissue engineering", Progress in Polymer Science. v. 38, n. 3-4, p. 584-671. 2013.

[8] MARQUES, D. R. "Fibras De Poli (Ácido Láctico-Co-Glicólico)/Poliisopreno Para Aplicação Em Engenharia De Tecidos", Tese de D.Sc., Programa de Pós-Graduação em Engenharia de Minas, Metalúrgica e de Materiais, Universidade Federal do Rio Grande do Sul, Porto Alegre, 2015. 
[9] PANDEY, A., PANDEY, G. C., ASWATH, P. B. "Synthesis of Polylactic AcidPolyglycolic Acid Blends Using Microwave Radiation", Journal of the Mechanical Behavior of Biomedical Materials, Arlington, p. 227-223. Dez. 2007.

[10] MARQUES, D. R. “Obtenção e Caracterização de Blendas Poliméricas de Poli (Ácido Láctico-coGlicólico) e Poliisopreno para Aplicação como Biomaterial”, Dissertação de M.Sc., Programa de PósGraduação em Engenharia de Minas, Metalúrgica e de Materiais, Universidade Federal do Rio Grande do Sul, Porto Alegre, 2011.

[11] REZENDE, C. A., DUEK, E. A. R. "Blendas de Poli (Ácido Lático-co-Ácido Glicólico)/Poli (Ácido Lático): Degradação in vitro", Polímeros: Ciência e Tecnologia, Sorocaba, pp. 36-44. Out. 2002.

[12] ISOTALO, T. M., et al., "Biocompatibility Properties of a New Braided Biodegradable Urethral Stent: a Comparison With a Biodegradable Spiral and a Braile Metallis Stent in The Rabbit Urethra", British Journal of Urology Internacional. v. 97. p. 856-859. 2005.

[13] CHEN, F., QIAN, J. "Studies on the thermal degradation of cis-1,4-polyisoprene q", Fuel, v. 81, p. 2071-2077. 2002.

[14] LIPATOV, Y. S. "Polymer blends and interpenetrating polymer networks at the interface with solids", Progress in Polymer Science, v. 27, n. 9, p. 1721-1801. 2002.

[15] GUASTALDI, A. C., APARECIDA, A. H. "Fosfatos de cálcio de interesse biológico: Importância como biomateriais, propriedades e métodos de obtenção de recobrimentos", Química Nova. v. 33, n. 6, p.13521358, 2010.

[16] SALINAS, A. J., VALLET-REGI, M. "Bioactive ceramics: from bone grafts to tissue engineering", $R S C a d v$. n. 28, p. 11116-11131. 2013.

[17] WEBSTER, T. J., ERGUN, C., DOREMUS, R.H., et al., "Specific proteins mediate enhanced osteoblast adhesion on nanophase ceramics", Journal of Biomedical Materials Research, v. 51, n. 3, p. 475- 483, 2000.

[18] MIZUNO, M., SHIOMI, Y., MINATO, K., et al., "Fucogalactan isolated from Sarcodon aspratus elicits release of tumor necrosis factor-a and nitric oxide from murine macrophages", Immunopharmacology, v.46, p.113-121, 2000.

[19] CHENG, K., WENG, W. Zinc-and Fluorine-Doped HA Coatings via Sol-Gel Method. In: ZHANG, S. (Ed.). Hydroxyapatite, Coatings for Biomedical Applications. New York, CRC Press, 2013. p. 145-200.

[20] MAHYUDIN F., WIDHIYANTO L., HERMAWAN, H. "Biomaterials in Orthopaedics", In: MAHYUDIN F., HERMAWAN, H. (Ed.) Biomaterials and Medical Devices: A Perspective from an Emerging Country. Cham: Springer, 2016. p. 161-181.

[21] FARIAS, E. S. et al., "Revestimento de superfícies de Titânio com Hidroxiapatita através de Sistema de Deposição Eletroforética”, Disciplinarum Scientia. Série: Naturais e Tecnológicas, Santa Maria, v. 18, n. 2, p. 65-77, 2017.

[22] MOTTA, A.C., DUEK, E.A.R. "Síntese, caracterização e degradação "in vitro" do poli (L-ácido lácticoco-ácido glicólico)”,Matéria (Rio J.), Rio de Janeiro, v. 11, n. 3, pp. 340-350, Sept. 2006.

[23] QUEIROZ, D. P. “Diagrama de Fases, Propriedades Térmicas e Morfológicas de Blendas de Poli (Ácido Láctico) e Poli (Metacrilato de Metila)”, Tese de D.Sc., Universidade Estadual de Campinas, Campinas, 2000.

[24] WU, Y., CLARK, R. "Electrohydrodynamic atomization: a versatile process for preparing materials for biomedical applications”, J. Biomater. Sci. Polymer Edn, v. 19, n. 5, p. 573-601, 2008.

[25] PEREA, G, N, R. "Eletrofiação de Nanocompósito de Poli(L-Ácido Lático) com Hidroxiapatita para Regeneração Óssea”, Dissertação de M.Sc., Programa de Pós-Graduação em Engenharia Mecânica, Faculdade de Engenharia Mecânica da Universidade Estadual de Campinas, Campinas, 2011.

[26] DÍEZ-PASCUAL, A.M., DÍEZ-VICENTE, A.L. "Multifunctional poly(glycolic acid-co-propylene fumarate) electrospun fibers reinforced with graphene oxide and hydroxyapatite nanorods", J Mater Chem B. v. 5, pp. 4084-96, 2017.

[27] XU, X., CHEN, X., LIU, A., et al., "Electrospun poly(L-lactide)-grafted hydroxyapatite/poly(L-lactide) nanocomposites fibres", Eur. Polym. J., v. 43, p. 3187-3196, 2007. 


\section{ORCID}

Pâmella Schramm Oliveira

https://orcid.org/0000-0001-6461-0367

Luiz Fernando Rodrigues Junior

https://orcid.org/0000-0002-5753-5503

Tiago Moreno Volkmer

https://orcid.org/0000-0002-1758-8504

Nayrim Brizuela Gerra

https://orcid.org/0000-0002-2873-0975

Luís Alberto Loureiro dos Santos

https://orcid.org/0000-0002-9099-9748 